\title{
Emergence of kinetic behavior in streaming ultracold neutral plasmas
}

\author{
P. McQuillen, J. Castro, S. J. Bradshaw, and T. C. Killian \\ Department of Physics and Astronomy and Rice Quantum Institute, Rice University, \\ Houston, Texas 77005, USA
}

(Received 27 January 2015; accepted 8 April 2015; published online 23 April 2015)

\begin{abstract}
We create streaming ultracold neutral plasmas by tailoring the photoionizing laser beam that creates the plasma. By varying the electron temperature, we control the relative velocity of the streaming populations, and, in conjunction with variation of the plasma density, this controls the ion collisionality of the colliding streams. Laser-induced fluorescence is used to map the spatially resolved density and velocity distribution function for the ions. We identify the lack of local thermal equilibrium and distinct populations of interpenetrating, counter-streaming ions as signatures of kinetic behavior. Experimental data are compared with results from a one-dimensional, two-fluid numerical simulation. (C) 2015 AIP Publishing LLC. [http://dx.doi.org/10.1063/1.4918705]
\end{abstract}

\section{INTRODUCTION}

The behavior and theoretical description of a plasma depends critically on the ratio of the mean free path for constituent particles to the length scale of plasma inhomogeneities $\left(|n / \nabla n|\right.$ for plasma density $n$, or $\left|T_{i, e} / \nabla T_{i, e}\right|$ for plasma ion or electron temperature $T_{i, e}$ ). For collisional systems in which the mean free path is very small, particles remain close to local thermal equilibrium, and transport and collective motions can be described with a hydrodynamic treatment. Because of the increasing mean free path with particle kinetic energy, the situation can already change for mean free path on the order of or longer than $1 \%$ of the length scale of plasma gradients. ${ }^{1}$ This gives rise to kinetic effects such as streaming plasmas and non-local transport, which are intensely studied because of their importance in many plasma environments, such as high-density laser-produced plasmas $^{1-18}$ and astrophysical plasmas like the solar wind, ${ }^{19}$ solar atmosphere, ${ }^{20,21}$ solar flares, ${ }^{22}$ and supernovae. ${ }^{23}$

Here, we show the emergence of kinetic behavior for ions in an ultracold neutral plasma ${ }^{24,25}$ by creating colliding plasma streams with controllable relative velocity and density. It is the first observation in ultracold neutral plasmas of ion transport ${ }^{26-30}$ that cannot be explained with a hydrodynamic treatment. The emergence of kinetic effects is heralded by the absence of local thermal equilibrium for ions and interpenetrating streaming plasma populations. This compliments recent work on acoustic-wave phenomena in ultracold neutral plasmas in the purely hydrodynamic regime. ${ }^{28-30}$ Results are compared with a two-fluid hydrodynamic code. ${ }^{31,32}$

\section{EXPERIMENTAL SETUP}

Ultracold neutral plasmas are created through photoionization of strontium atoms from a magneto optical trap (MOT) as described in Ref. 33. The initial electron temperature is tunable $\left(T_{e}(0) \approx 1-1000 \mathrm{~K}\right)$ and is determined by the excess energy of the ionizing photons above threshold, $T_{e}=\left(2 / 3 k_{B}\right) \Delta E_{i o n}$, which is calibrated by a combination of Rydberg spectroscopy and absorption spectroscopy in an indium vapor cell. The electron temperature is confirmed by fitting the evolution of the plasma density distribution. ${ }^{27}$ Ion temperatures are set to approximately $1 \mathrm{~K}$ by disorderinduced heating, ${ }^{34,35}$ resulting in strongly coupled ions in the liquid-like regime. ${ }^{25,36}$ The plasma density distribution is a spherical Gaussian, $n(r)=n_{0} \exp \left(-r^{2} / 2 \sigma_{0}^{2}\right)$, with $n_{0} \sim 6 \times 10^{14} \mathrm{~m}^{-3}$ and $\sigma_{0} \sim 1.5 \mathrm{~mm}$. Typical plasma evolution is dominated by the cloud's adiabatic expansion into the surrounding vacuum which is driven by gradients in the electron thermal pressure on a time scale of $\approx 10 \mu \mathrm{s}^{27}$

A gap in the center of the plasma is created by placing an opaque wire in the path of the ionizing beam, which results in two plasma hemispheres that stream into each other during plasma expansion. Streaming plasmas are precursors to complex phenomena such as instabilities, shock waves, and solitons, 9,16,37,38 and they have been studied in a wide variety of environments such as the solar wind, ${ }^{39}$ supernovae, ${ }^{23}$ double plasma devices, ${ }^{40-43}$ and laser-produced plasmas. $^{2-6,9,16}$ A partially transmitting strip, instead of an opaque wire, was recently used to create a smaller amplitude density depletion in the plasma center. ${ }^{30}$ This led to hydrodynamic phenomena such as gap splitting and propagation of localized density depletions at the ion acoustic wave speed. A similar technique was used to create periodic density modulations and excite ion acoustic waves. ${ }^{28}$

As a diagnostic, a tuneable, narrowband laser induces fluorescence from the primary $\mathrm{Sr}^{+}$transition, ${ }^{2} \mathrm{~S}_{1 / 2}-{ }^{1} \mathrm{P}_{1 / 2}$ at $\lambda=422 \mathrm{~nm}^{27}$ This excitation beam is masked by a $1 \mathrm{~mm}$ slit to illuminate only a central sheet of the plasma. ${ }^{44}$ Fluorescence emitted close to perpendicular to the plane of the ionizing and $422 \mathrm{~nm}$ beams is imaged onto an intensified CCD camera with a pixel size of $52 \mu \mathrm{m}$. The resulting image, $F(x, y, \nu)$, is frequency dependent due to the natural linewidth and Doppler-broadening of the transition. ${ }^{44}$ Images are combined to obtain a map of the density distribution of the ions in the illuminated plane, $n_{i}(x, y, z \approx 0)$ or the spatially resolved, distribution function for velocity perpendicular to the gap. ${ }^{28,44}$

We also model the plasma evolution with a onedimensional two-fluid simulation ${ }^{31,32}$ in which we treat 
bulk flows (transport, compression, and rarefaction), energy exchange between particle species by collisions and the work done by small-scale electric fields. We found that thermal conduction and viscous interactions made negligible contributions to the plasma energy balance in the temperature regimes explored here. The initial density distribution is matched to the profile observed experimentally within $100 \mathrm{~ns}$ of plasma creation. Ion temperature is set equal to the value after disorder-induced heating ${ }^{35}$ measured with LIF spectroscopy. ${ }^{44}$ A one-dimensional simulation will not correctly reproduce the adiabatic cooling of electrons and ions caused by the three dimensional expansion of the ultracold plasma. ${ }^{27}$ But the deviation is small and does not affect the phenomena discussed here.

The critical parameter that demarcates hydrodynamic (collisional) and kinetic (collisionless) regimes is the ratio between the mean free paths of the particles and a characteristic scale-length of the plasma (the Knudsen number). When the mean free path is small compared with this scale length then the plasma is collisional and the particle velocity distribution functions are near Maxwellian. When the meanfree-path exceeds the characteristic scale-length then kinetic phenomena can become important.

Mean free paths and collision timescales for ion-ion and electron-electron collisions, respectively, for conditions relevant to strontium ultracold plasmas are defined in terms of the generalized collision frequency for a test particle $(\alpha)$ streaming with speed $v_{\alpha}$ through a background of field particles $(\beta)$ with and mass $m_{\alpha, \beta}$ (Ref. 45)

$$
\nu^{\alpha, \beta}=\left(1+m_{\alpha} / m_{\beta}\right) \psi\left(x^{\alpha, \beta}\right) \nu_{0}^{\alpha, \beta},
$$

where

$$
\begin{gathered}
\psi(x)=\frac{2}{\sqrt{\pi}} \int_{0}^{x} t^{1 / 2} \exp (-t) d t, \\
x^{\alpha, \beta}=\frac{m_{\beta} v_{\alpha}^{2}}{2 k_{B} T_{\beta}},
\end{gathered}
$$

and

$$
\nu_{0}^{\alpha, \beta}=\frac{e_{\alpha}^{2} e_{\beta}^{2} \lambda_{\alpha, \beta} n_{\beta}}{4 \pi \epsilon_{0}^{2} m_{\alpha}^{2} v_{\alpha}^{3}} .
$$

$\lambda_{\alpha, \beta}$ is the Coulomb logarithm for the interaction. In the absence of a gap, at the density of the unperturbed plasma, the ion-ion mean free path for ions with four times the thermal speed for $T_{i}=1 \mathrm{~K}$ is $40 \mu \mathrm{m}$ for screening by $100 \mathrm{~K}$ electrons. This is small compared to the plasma size $\sigma_{0}$, and we expect predominantly hydrodynamic behavior for the ion motion such as overall plasma expansion. Electron mean free paths are much longer, which might become important in higher temperature plasmas where the streaming population could influence the energy balance and, consequently, the dynamics of the plasma via high order moments of the distribution function, such as the heat flux. However, for the parameter space and phenomena explored here, we are not sensitive to these effects.

\section{RESULTS AND DISCUSSION}

A qualitative understanding of the plasma dynamics when a gap is formed can be gained from looking at the evolution of the ion density distributions. Figure 1 shows data for conditions that display phenomena characteristic of the crossover regime from hydrodynamic to kinetic $\left(T_{e}(0)=25 \mathrm{~K}\right)$ and the fully kinetic regime $\left(T_{e}(0)=105 \mathrm{~K}\right)$. The initial density distributions are similar for both cases. Figures 1(a)-1(c) show false color images of the twodimensional central density slice $n_{i}(x, y, z \approx 0)$, and Figs. 1(d) and 1(e) show 1D central density traces at various times for initial electron temperature (d) $T_{e}(0)=25 \mathrm{~K}$ and (e) $T_{e}(0)=105 \mathrm{~K}$. (Due to optical access constraints, the plasma is slightly larger than the region illuminated by the fluorescence beam, creating the apparent oblong shape in the $2 \mathrm{D}$ images.)

Immediately after plasma formation, a gap is evident (Figs. 1(a), 1(d), and 1(e)) with a length scale for the sides of the gap of about $l \equiv|n / \nabla n| \sim 400 \mu \mathrm{m}$, which is determined by diffraction of the ionizing beam and is much less than $\sigma_{0}$. The subsequent movement of ions into the gap can be understood hydrodynamically using the coupled ion-electron momentum balance equations. ${ }^{26}$ In the presence of any density gradient, ions are accelerated $\left(a_{0}\right)$ by the electron pressure $\left(P_{e}\right)$ according to $M n a_{0} \approx-\nabla P_{e} \approx-\nabla\left(n k_{B} T_{e}(0)\right) . T_{e}(0)$ is expected to be close to uniform, ${ }^{46}$ and a characteristic ion velocity after acceleration is $v_{f} \approx \sqrt{k_{B} T_{e}(0) / M}$. This suggests a timescale for motion of

$$
\tau \approx \frac{v_{f}}{a_{0}} \approx-\frac{n}{\nabla n} \sqrt{\frac{M}{k_{B} T_{e}(0)}} .
$$

At the sides of the gap, this timescale is fast and can be identified with the timescale for ion streams to form and interact in the center of the gap, $\tau_{\text {fast }} \approx \sqrt{M l^{2} /\left[k_{B} T_{e}(0)\right]}$. Here, $\tau_{\text {fast }} \sim 8 \mu$ s for $T_{e}(0)=25 \mathrm{~K}$ and $\tau_{\text {fast }} \sim 4 \mu \mathrm{s}$ for $T_{e}(0)=105 \mathrm{~K}$, which approximately matches the timescales seen in the experiment. Note that this argument also explains the overall expansion of the plasma, for which the length scale is that of the spherical Gaussian, $-n / \nabla n \approx \sigma_{0}$. This gives the characteristic expansion time of a self-similar expansion, $\tau_{\text {exp }} \approx \sqrt{M \sigma_{0}^{2} /\left[k_{B} T_{e}(0)\right]}\left(\right.$ Ref. 25) $\left(\tau_{\text {exp }}=15 \mu\right.$ s and $31 \mu \mathrm{s}$ for $T_{e}(0)=25 \mathrm{~K}$ and $T_{e}(0)=105 \mathrm{~K}$, respectively).

\section{A. Signatures of the kinetic regime in the density evolution}

Whether the ion streams behave hydrodynamically or kinetically when they collide depends upon the inter-stream mean free path at the confluence. The relevant densitygradient length scale for this interaction can be approximated as something between the length scale of the side of the gap ( $l$ ) and the gap width. For short mean free path, the streams cannot penetrate, and a density enhancement emerges at the confluence. The gap splits and two density depletions propagate away from plasma center as seen in Ref. 30 . There is indication of this behavior for $T_{e}(0)=25 \mathrm{~K}$ (Figs. 1(b) and 1(c)). However, the central feature never rises to the height 

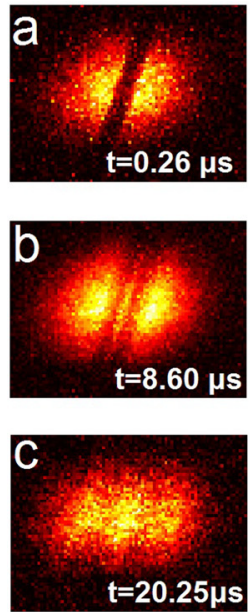
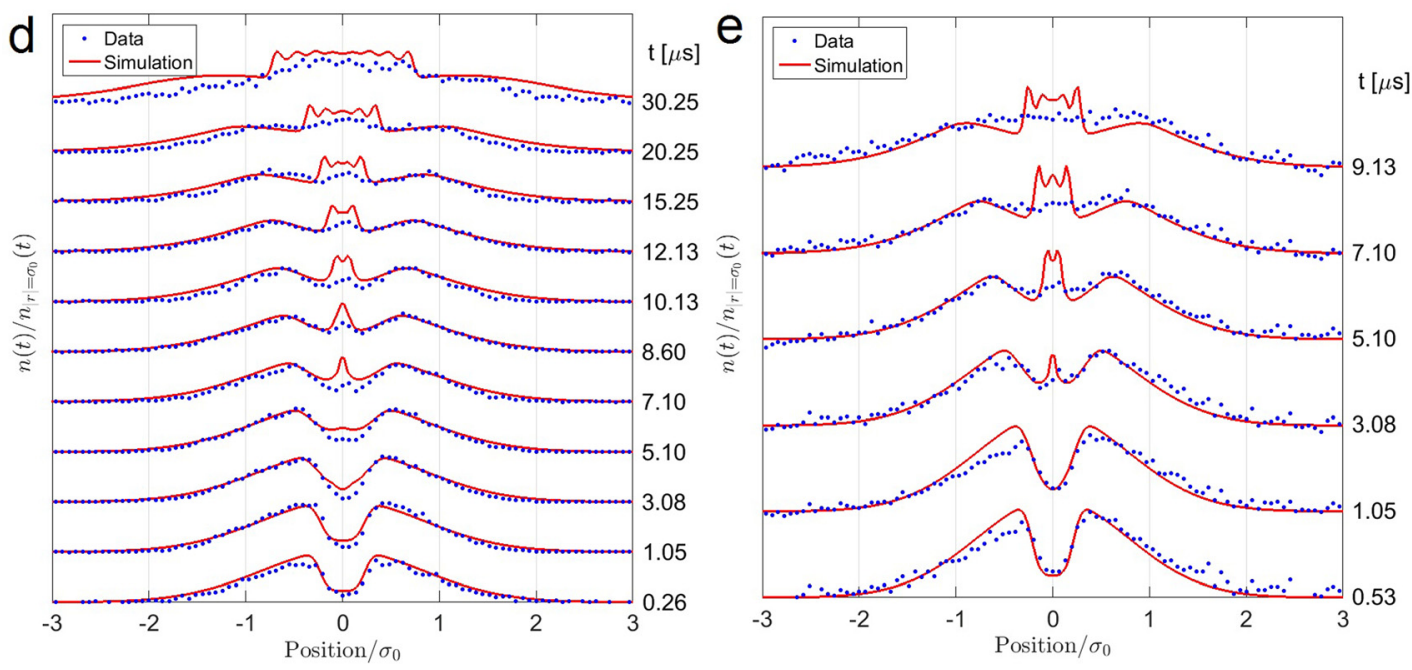

FIG. 1. Evolution of the ion density distributions. The time since ionization is indicated. Initial peak densities are $6 \times 10^{14} \mathrm{~m}^{-3}$. (a)-(c) False color plots of the density distribution in the crossover between hydrodynamic and kinetic regimes $\left(T_{e}(0)=25 \mathrm{~K}\right)$. (d) $1 \mathrm{D}$ slices through the density profile for the same data as (a)-(c), normalized and offset for clarity. Note the emergence of the central feature as the gap splits. The hydrodynamic simulation overestimates the prominence of the central density enhancement and the density depletions propagating away from the gap region. (e) Same as (d) but for $T_{e}=105 \mathrm{~K}$ showing more kinetic behavior. Note the absence of a significant central feature at the confluence of the ions streaming into the gap and the poor agreement between the data and hydrodynamic simulation. Distance is normalized to plasma size $\sigma_{0}=1.5 \mathrm{~mm}$.

that the plasma would have had in the absence of an initial gap, and the amplitude of the features moving away from the center are much smaller than the initial gap (Fig. 1(d)). These two caveats indicate that the colliding streams in the $T_{e}(0)=25 \mathrm{~K}$ plasma are not fully in the hydrodynamic regime and kinetic effects are important. Experiments in Ref. 30 accessed and characterized the purely hydrodynamic regime for $T_{e}(0)=25 \mathrm{~K}$ by starting with a very small density depletion and approximately five times greater density in the gap. In this case, the central feature rose to full height, and the amplitude of the traveling density depletions showed no significant attenuation. Data from Ref. 30 are reproduced in Fig. 2 for comparison.

To estimate the mean free path $\ell_{m f p}$ for an ion in one stream moving through the other, we use the density in the center at time $\sim \tau_{\text {fast }} / 2 \sim 4 \mu \mathrm{s}$, which is about $10^{14} \mathrm{~m}^{-3}$, and take the velocity as $v_{f} \approx 50 \mathrm{~m} / \mathrm{s}$. Equation (4) yields $\ell_{m f p}=600 \mu \mathrm{m}$.
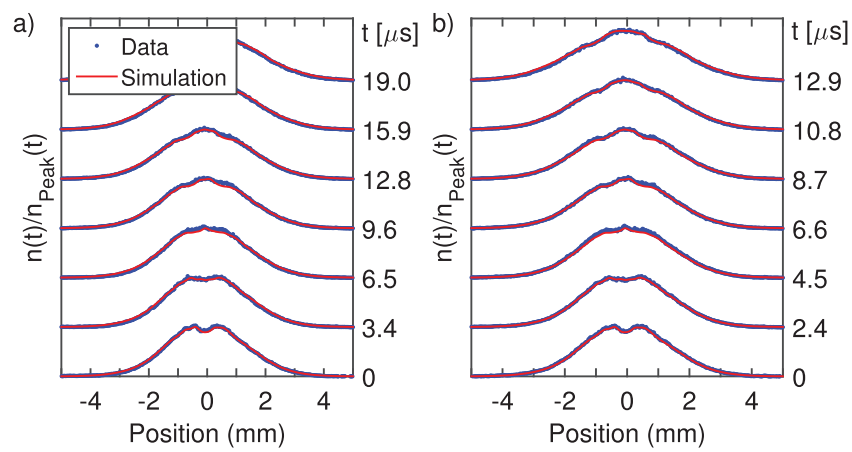

FIG. 2. Evolution of small-amplitude density perturbations that are well described by the hydrodynamic simulation. Initial electron temperatures are (a) $T_{e}(0)=25 \mathrm{~K}$ and (b) $T_{e}(0)=55 \mathrm{~K}$. Initial peak densities are $1 \times 10^{15} \mathrm{~m}^{-3}$. This data were previously published in Ref. 30, and it is shown here to highlight deviations from the hydrodynamic model evident in Fig. 1.
To highlight the difference between the density evolution in the experiments and what one would predict for hydrodynamic behavior, we show in Fig. 1(d) the results of a 1-D hydrodynamic simulation of the experimental data. For the hydrodynamic plasmas studied in Ref. 30, the simulation accurately captured all the features seen in the experiment, as demonstrated in Fig. 2. In Fig. 1, there is obvious discrepancy between experiment and the hydrodynamic simulation in the gap region. The simulation overestimates the prominence of the central feature and traveling depletions.

The mean free path is further increased by increasing the electron temperature and relative velocity of the colliding streams, as shown in Fig. 1(e) for $T_{e}(0)=105 \mathrm{~K}$. Making the same approximations as before yields $\ell_{m f p}=7 \mathrm{~mm}$. In this case, the hydrodynamic phenomena related to the gap (central density enhancement and traveling density depletions) are barely visible, indicating the dominance of kinetic effects. There is also very large discrepancy between experiment and the hydrodynamic simulation in the gap region.

\section{B. Measuring the velocity distribution function}

To probe the effect of the gap on the plasma dynamics more quantitatively and to support our statements regarding the emergence of kinetic behavior, we analyze the fluorescence excitation spectrum. We obtain the spectrum of a region of the plasma, $S(\nu)_{\text {region }}$, by summing the fluorescence from that region,

$$
\sum_{x, y \in \text { region }} F(x, y, \nu) \propto S(\nu)_{\text {region }} .
$$

We will first discuss the spectrum of the entire illuminated plasma sheet, which is obtained by expanding the region to encompass the entire field of view. Then we will discuss the spectra of resolved regions of the plasma near the gap. 
The spectral shape is dominated by Doppler shifts and broadenings resulting from ion velocities, and for simplicity we can neglect the small broadening of the spectra due to the natural linewidth of the transition. This allows us to interpret the spectrum as the distribution of ion velocities perpendicular to the gap by plotting the spectra versus velocity, where velocity is calculated as $\mathrm{v}=\delta \nu \lambda . \delta \nu$ is the detuning of the fluorescence excitation laser from resonance and $\lambda$ is the wavelength. (The FWHM, natural linewidth of the transition $\gamma / 2 \pi=20 \mathrm{MHz}$ leads to a width in velocity of $\delta \mathrm{v}=\gamma \lambda / 2 \pi=8.4 \mathrm{~m} / \mathrm{s}$.)

\section{Velocity distribution of the entire plasma}

Using this approximation, Fig. 3(a) shows the velocity distribution for the entire plasma sheet, which reflects directed ion motion (plasma expansion or streaming) and thermal ion motion. At early times, the distribution is unaffected by the existence of the gap, and the width reflects the ion temperature after disorder-induced heating (DIH). ${ }^{34,35,47}$ DIH occurs as excess potential energy in the initially random ion spatial distribution is converted to ion kinetic energy. The timescale for DIH is the inverse of the ion plasma oscillation frequency, $1 / \omega_{p i}=\sqrt{M \epsilon_{0} / n e^{2}} \sim 1 \mu \mathrm{s}$, where $M$ is the ion mass. This is much faster than the hydrodynamic timescale on which the plasma reacts to density inhomogeneities. After DIH, ion velocities increase as electron thermal energy is converted to directed ion motion through the hydrodynamic process discussed above. The distribution for a plasma without a gap broadens as the ions accelerate radially, but it retains a generally featureless Gaussian shape characteristic of a self-similar expansion. ${ }^{27}$

The spectrum of the perturbed plasma broadens more quickly and develops a bimodal shape. We will show below that bimodality reflects ions from the two sides of the gap streaming into the gap and through each other. This motion is still driven by the conversion of electron thermal energy

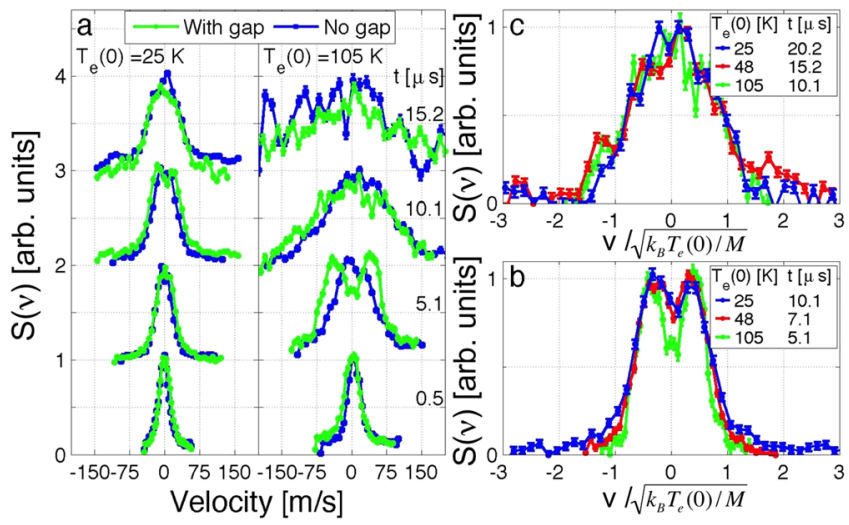

FIG. 3. Spectra of entire plasma sheet. (a) Effect of the gap and changing $T_{e}(0)$. Frequency has been converted to velocity (v) through the Doppler shift $\mathrm{v}=\nu / \lambda$, and time since ionization is indicated. Note the emergence of bimodal structure, corresponding to streaming plasmas. (b) Spectra of plasmas with gaps with scaled velocity and $t / \tau_{\text {fast }} \sim 1.3$. The universal behavior highlights the emergence of the new timescale, $\tau_{\text {fast }}$. (c) Same as (b) but for $t / \tau_{\text {exp }} \sim 0.7$. At later times, structure has vanished, and universal behavior indicates the scaling of the transfer of electron energy into plasma expansion velocity. into ion expansion energy, but it occurs on the timescale $\tau_{\text {fast }}$. Figure 3(b) shows spectra for the perturbed plasma at the same scaled time, $t / \tau_{\text {fast }} \sim 1.3$ and with velocity scaled by $v_{f}$. The universality of the scaled data emphasizes how the gradient in the electron thermal pressure drives the dynamics. Note that at later times (Fig. 3(a) top panel) the distributions for the perturbed and unperturbed plasmas display the same scaled width with no sign of bimodality. The structure in the global velocity distribution has vanished even though some structure in the density distribution still persists (Fig. 1).

\section{Spatially resolved velocity distributions and streaming plasmas}

To probe the local dynamics of the plasma, we analyze spectra and extract the 1D velocity distributions of small spatial regions in the vicinity of the gap, which measure local ion velocities and temperatures. ${ }^{27,44,48}$ Regions are arranged as shown in Fig. 4. Results in Figs. 5 and 6 compare regional spectra evolution for two different initial electron temperatures, $25 \mathrm{~K}$ and $105 \mathrm{~K}$, respectively. Spectra are obtained from Eq. (6). We only show the spectra of the central region and the regions to the right of the gap since due to the symmetry of our plasma with respect to the gap, corresponding regional spectra in the two hemispheres mirror each other. The shift of a distribution represents the mean velocity of an ion population, and the width represents the rms velocity

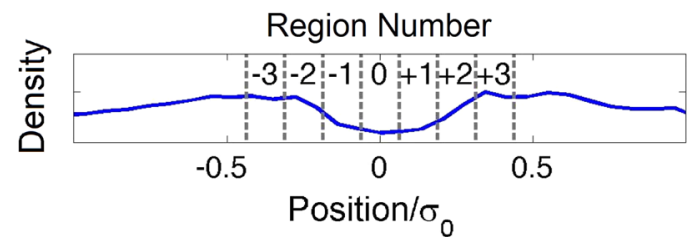

FIG. 4. Static regions used in analysis of local ion velocities and temperatures are shown relative to the gap in the initial density profile. The dimensions of each regions are $190 \mu \mathrm{m}$ perpendicular to the gap and $760 \mu \mathrm{m}$ parallel to it.

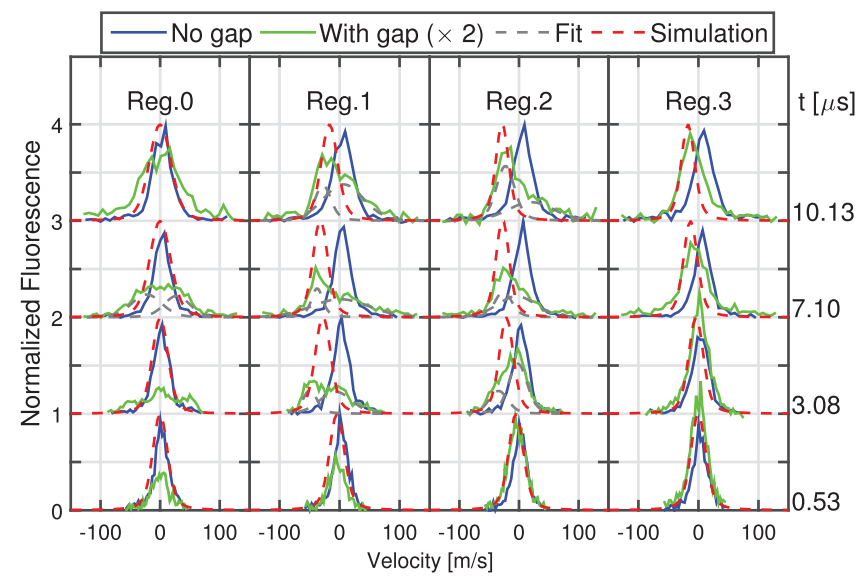

FIG. 5. Spatially-resolved regional spectra for plasmas with $T_{e}(0)=25 \mathrm{~K}$. Time since ionization is indicated on the right. The solid lines are data with and without the gap while dashed lines are fits to perturbed velocity distributions. Shifts represent ion mean velocities and widths represent ion temperature. For plasmas without the gap, positive velocities reflect expansion. ${ }^{44}$ For plasmas with the gap, multiple populations appear moving left and right. The dotted lines show results of the hydrodynamic simulation. 


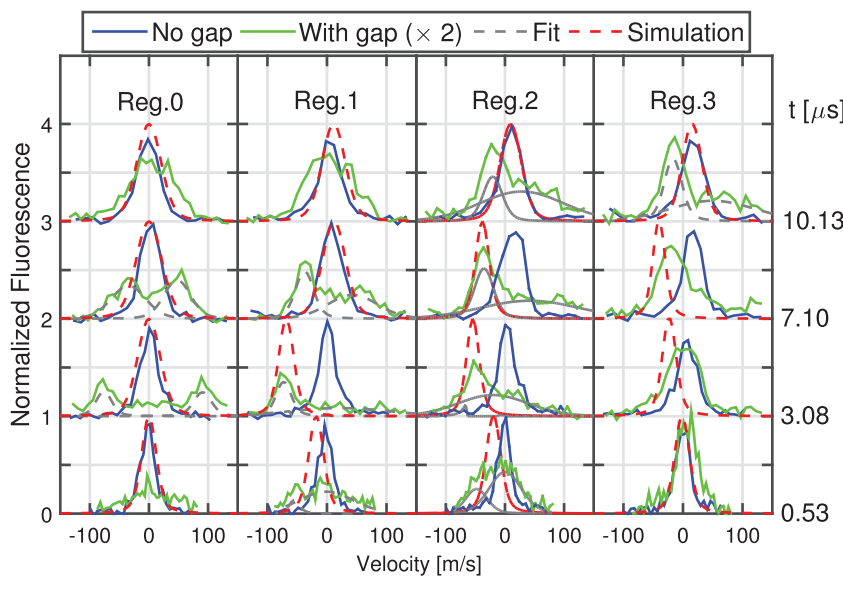

FIG. 6. Same as Fig. 5, but for $T_{e}(0)=105 \mathrm{~K}$. Compared to Fig. 5, in the perturbed plasma, average velocities are much higher and populations moving to the right are more prominent.

spread. ${ }^{44}$ We typically quote the width in terms of an effective local temperature, but the width is also affected by spread in average ion velocity across the region and lack of thermal equilibrium.

Without the gap, regional spectra show expansion velocities that increase with time and distance from center as expected. ${ }^{25}$ For regions far from the gap and especially at early times, the distribution shape, shift, and width are essentially unchanged by introduction of a gap. But there are dramatic differences between spectra of perturbed and unperturbed plasmas for inner regions.

Signal at positive velocities represent ions moving away from plasma center, whereas negative velocities represent ions moving towards it. Positive and negative velocity groups in region 0 indicate ions moving through the center of the gap. It is important to note that ions move through the regions, so spectra for a given region at different times may reflect different ions. For instance, neglecting collisions and given the dimension of the regions, particles in a particular region with mean velocity of $60 \mathrm{~m} / \mathrm{s}$, will be in a contiguous region in approximately $\sim 3 \mu \mathrm{s}$.

There are many common features in $T_{e}(0)=25 \mathrm{~K}$ and $T_{e}(0)=105 \mathrm{~K}$ data that illustrate the general behavior of streaming ultracold neutral plasmas. At early times $(0-3 \mu \mathrm{s})$, the density gradient in regions 1 and 2 produces an acceleration of ions towards the gap. This is followed by the appearance of two beams of high velocity ions crossing each other in region 0 . The stream velocities closely match the characteristic velocity introduced earlier $\left(v_{f}=\sqrt{k_{B} T_{e}(0) / M}=100 \mathrm{~m} / \mathrm{s}\right.$ and $50 \mathrm{~m} / \mathrm{s}$ for $105 \mathrm{~K}$ and $25 \mathrm{~K}$, respectively). The bulk velocity of each population greatly exceeds the ion thermal speed and is comparable to the phase velocity of ion acoustic waves. At later times $(7-10 \mu \mathrm{s})$, these populations have crossed region 0 , and ions that originated on the other side of the gap now appear in regions $1-3$ as ions moving away from the gap. These interpenetrating streams and local velocity distributions that cannot be defined by a single Maxwellian with a well-defined temperature are indicators that the plasma is in the kinetic regime. Data from hydrodynamic plasmas studied in Ref. 30 showed no deviation from local Maxwellians.

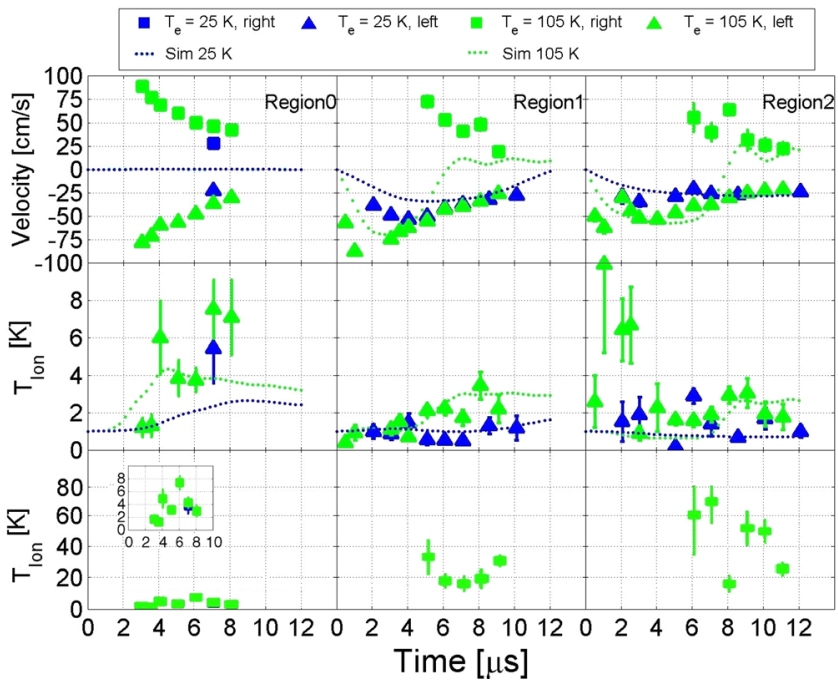

FIG. 7. Ion velocities and temperatures for the fast moving streams in Figs. 6 and 5. (top) Velocities of the fast moving streams. Note that streams moving towards the gap (velocity $<0$ ) appear first in region 2 . Streams moving away from the gap velocity $>0$ ) appear first in region 0 . (middle) Temperature of left-moving streams moving towards the gap. (bottom) Temperature of right-moving streams that have crossed the gap and are moving away from it. Note the different temperature scales.

In Figs. 5 and 6, it is interesting to note that ion populations remain cold until they have passed through the gap, after which their velocity spread greatly increases (Fig. 7). Ions remaining in the gap or moving in at later times $(10 \mu \mathrm{s})$ eventually merge into one population of hot ions.

Important differences between the data sets reflect the variation in electron thermal pressure. For example, moving populations appear sooner for $T_{e}(0)=105 \mathrm{~K}(0.5 \mu \mathrm{s})$ than $T_{e}(0)=25 \mathrm{~K}$, and velocities are higher for higher $T_{e}(0)$ (Fig. 7). The ion temperature in region zero at later times is also hotter for $T_{e}(0)=105 \mathrm{~K}$ than $T_{e}(0)=25 \mathrm{~K}$.

There is also significant difference in the penetration depth of ions from one side into the plasma on the other side of the gap. We estimate the penetration length in the plasma from the farthest excursion of right-moving populations. For $T_{e}(0)=105 \mathrm{~K}$, right-moving ions clearly appear in region 3 $(10 \mu \mathrm{s})$, while for $T_{e}(0)=25 \mathrm{~K}$ right-moving ions appear in region 1 , and perhaps region 2 , and the population is less distinct than for $T_{e}(0)=105 \mathrm{~K} . T_{e}(0)=48 \mathrm{~K}$ (not shown) displays intermediate behavior. No clear right-moving population can be found at times later than $\sim 10 \mu \mathrm{s}$ for any $T_{e}(0)$ (data not shown). One expects a greater penetration length for higher $T_{e}(0)$ because the Coulomb collision cross section decreases with collision velocity, $\sigma \propto 1 / v^{4}$. From these measurements, it should also be possible to estimate the stopping power of electrons and strongly coupled ions in an ultracold plasma for a penetrating ion stream. ${ }^{49-52}$

Hydrodynamic simulations are included in Figs. 5 and 6 to emphasize the breakdown of the hydrodynamic approximation. But it is also interesting to note the nature of the discrepancy between the data and the simulation. In the central region, when two symmetric, relatively cold, streaming plasma populations are present, the hydrodynamic code predicts a single cold population with zero average velocity, which is what one would expect given the constraint in the 
simulation of a local thermal equilibrium for the ions. However, in regions away from the center, the distributions from the hydrodynamic simulation are close the distribution for the population of ions that have not crossed the gap, but the contributions from ions that have streamed across the gap are absent in the hydrodynamic simulation. This reflects the inability of the hydrodynamic code to account for nonlocal transport. The hydrodynamic code also tends to underestimate the ion temperature at later times.

\section{E. Trends in the ion temperature and bulk velocity}

To accurately extract ion temperatures and velocities we fit regional spectra (Figs. 6 and 5) to one or the sum of two Voigt profiles. The Voigt profile is the convolution of a Lorentzian with homogeneous width $\gamma$ and shifted Gaussian for Doppler effects. ${ }^{25}$ In this way, we take spectral broadening due to the natural linewidth of the transition into account for quantitative analysis. As mentioned above, the temperature is extracted from the width of the Gaussian. Temperature measurements are sensitive to many systematic effects, but it still an informative parameter. Data that fit well to two profiles give clear indication of different populations of streaming ions with different mean velocities and temperatures. In these cases, the dotted lines show the individual Voigt profiles of each population, and the sum of both these curves is in good agreement with the data.

Ion velocities and temperatures for the streams are shown in Fig. 7 whenever they are resolvable. For higher $T_{e}(0)$, we obtain faster streaming velocities, which is consistent with the separation of the bimodal distribution in Fig. 3(a). In regions 1 and 2, populations that are moving into the gap (negative velocities and middle panel) appear to be relatively cold $\left(T_{i} \lesssim 2 \mathrm{~K}\right)$, while populations that have traveled across the gap and moved an appreciable distance through a counter-streaming plasma (positive velocities and bottom panel), have higher temperatures $\left(T_{i} \gtrsim 20 \mathrm{~K}\right)$. In the center, region 0 , particles moving to the left and right show very similar ion temperatures.

For all regions, velocities decrease with time. Decreasing plasma gradient and decreasing $T_{e}$ (Ref. 27) with time imply that ions moving through the gap at later times have experienced less acceleration during their trajectories than at early times. But velocities also decrease for an individual population as it moves through the regions. This may reflect that after ions move past the gap, the local electric field opposes the average motion for that population. Damping or collisions may also play a role, which is supported by the considerably higher temperatures measured for populations that have crossed the gap.

When only one distinct population of ions is visible in the velocity distribution function, such as in region 1 at early times $(<3 \mu \mathrm{s})$, the hydrodynamic simulation does a reasonable job of predicting the average ion velocity (7(top)). When two streaming populations are present, however, such as in region 1 at later times or in region 0 , the bimodal velocity distribution is far outside the regime that can be treated by a single Maxwellian distribution hydrodynamic treatment. The hydrodynamic approximation of a single ion population with well-defined temperature and average velocity results in a velocity distribution that tends to track the average velocity. In the central region, for example, the average velocity is always zero. Similarly, in regions farther from the gap (region 2), the temperature in the simulation agrees reasonably well with the temperature of the population moving towards the gap (Fig. 7 (middle)), indicating these ions are relatively unaffected by the small population of high velocity ions that have crossed the gap and are streaming through the region. This highlights the phenomena that characterize departure from the hydrodynamic regime.

\section{CONCLUSION}

In this work, we have used the ability to create streaming populations of particles in ultracold neutral plasmas to demonstrate the crossover from hydrodynamic to kinetic behavior and predominantly kinetic behavior. This work is complimentary to a recent paper in which we studied plasmas with a similar initial geometry in the hydrodynamic regime. ${ }^{30}$ The ratio of the ion mean free path to the length scale of plasma inhomogeneities as ions move through the gap and the populations collide is the crucial parameter for determining the regime of behavior, and it can be tuned by varying the ion density in the gap and the electron temperature, which changes the stream velocity. The hydrodynamic regime is characterized by a density enhancement at the confluence of the plasma streams, gap splitting, and localized density depletions that propagate away from the initial gap. The kinetic regime lacks these features and displays velocity distributions that strongly deviate from local thermal equilibrium and show distinct populations of interpenetrating, counter-streaming plasmas.

There are many future directions in which this work can go. This study has focused on a qualitative description of plasma behavior in the various regimes, but it would be informative to quantitatively map the boundary between different regimes in the parameter space of stream velocity and plasma density in the gap. One could use, for example, the appearance of streaming populations or visibility of a localized density enhancement in the confluence region as an indicator of the plasma regime.

A full kinetic description will allow a much more detailed and quantitative discussion of the observed effects. Alternatively, data such as this can provide a stringent test of kinetic codes. Kinetic codes, however, are much more computationally intensive. Rather than move to a full kinetic description, a common strategy is to generalize transport coefficients in order to extend the usefulness of hydrodynamic treatments. This is often done to treat nonlocal transport in plasmas with low collisionality, in which particle and energy fluxes at a given location are affected by conditions in distant regions of the plasma, which represents an important and complex problem. Often, ad hoc schemes are used in which the flux is simply limited to a fraction of its freestreaming value. ${ }^{1,8,13,53-55}$ Data such as presented here can provide a measurement of fluxes and thermodynamic gradients with high temporal and spatial resolution. This could provide a test of flux-limiting treatments or inform improved 
schemes to extend hydrodynamic models to address nonlocal effects.

We have mentioned that it should be possible to estimate the stopping power of ions by studying the penetration depth of the ion streams. Away from the initial gap, the streams move into regions of cold ions that are strongly coupled, which gives greater interest to such a measurement. ${ }^{49-52}$ With sharper density features in the initial plasma, it may also be possible to excite and study shock waves. ${ }^{46,56}$

\section{ACKNOWLEDGMENTS}

This work was supported by the National Science Foundation (PHY-0714603). S.J.B. acknowledges funding support from NASA.

${ }^{1}$ D. R. Gray and J. D. Kilkenny, Plasma Phys. 22, 81 (1980).

${ }^{2}$ K. Papadopoulos, R. C. Davidson, J. M. Dawson, I. Haber, D. A. Hammer, N. A. Krall, and R. Shanny, Phys. Fluids 14, 849 (1971).

${ }^{3}$ D. W. Koopman, Phys. Fluids 14, 1707 (1971).

${ }^{4}$ S. O. Dean, E. A. McLean, J. A. Stamper, and H. R. Griem, Phys. Rev. Lett. 27, 487 (1971)

${ }^{5}$ J. B. McBride, E. Ott, J. P. Boris, and J. H. Orens, Phys. Fluids 15, 2367 (1972).

${ }^{6}$ A. Y. Cheung, R. R. Goforth, and D. W. Koopman, Phys. Rev. Lett. 31, 429 (1973).

${ }^{7}$ J. F. Luciani, P. Mora, and R. Pellat, Phys. Fluids 28, 835 (1985).

${ }^{8}$ J. Albritton, E. Williams, I. Bernstein, and K. Swartz, Phys. Rev. Lett. 57, 1887 (1986).

${ }^{9}$ A. R. Bell, P. Choi, A. E. Dangor, O. Willi, D. A. Bassett, and C. J. Hooker, Phys. Rev. A 38, 1363 (1988).

${ }^{10}$ S. A. Uryupin, S. Kato, and K. Mima, Phys. Plasmas 2, 3100 (1995).

${ }^{11}$ V. Y. Bychenkov, W. Rozmus, V. T. Tikhonchuk, and A. V. Brantov, Phys. Rev. Lett. 75, 4405 (1995).

${ }^{12}$ G. P. Schurtz, P. D. Nicolai, and M. Busquet, Phys. Plasmas 7, 4238 (2000).

${ }^{13}$ G. Gregori, S. H. Glenzer, J. Knight, C. Niemann, D. Price, D. H. Froula, M. J. Edwards, and R. P. J. Town, Phys. Rev. Lett. 92, 205006 (2004)

${ }^{14}$ A. V. Brantov, V. Y. Bychenkov, and W. Rozmus, Plasma Phys. Rep. 32, 337 (2006).

${ }^{15}$ P. D. Nicolaï, J.-L. Feugeas, and G. P. Schurtz, Phys. Plasmas 13, 032701 (2006).

${ }^{16}$ L. Romagnani, S. V. Bulanov, M. Borghesi, P. Audebert, J. C. Gauthier, K. Löwenbrück, A. J. Mackinnon, P. Patel, G. Pretzler, T. Toncian et al., Phys. Rev. Lett. 101, 025004 (2008).

${ }^{17}$ Z. Zheng, W. Rozmus, V. Y. Bychenkov, A. V. Brantov, and C. E. Capjack, Phys. Plasmas 16, 102301 (2009).

${ }^{18}$ A. Marocchino, M. Tzoufras, S. Atzeni, A. Schiavi, P. D. Nicola, J. Mallet, V. Tikhonchuk, and J.-L. Feugeas, Phys. Plasmas 20, 022702 (2013).

${ }^{19}$ D. Perrone, R. O. Dendy, I. Furno, R. Sanchez, G. Zimbardo, A. Bovet, A. Fasoli, K. Gustafson, S. Perri, P. Ricci et al., Space Sci. Rev. 178, 233 (2013).

${ }^{20}$ S. J. Bradshaw and J. Raymond, Space Sci. Rev. 178, 271 (2013).

${ }^{21}$ M. J. West, S. J. Bradshaw, and P. J. Cargill, Solar Phys. 252, 89 (2008).

${ }^{22}$ J. T. Karpen and C. R. DeVore, Astrophys. J. 320, 904 (1987).

${ }^{23}$ C. Courtois, R. A. D. Grundy, A. D. Ash, D. M. Chambers, N. C. Woolsey, R. O. Dendy, and K. G. McClements, Phys. Plasmas 11, 3386 (2004).
${ }^{24}$ T. C. Killian, S. Kulin, S. D. Bergeson, L. A. Orozco, C. Orzel, and S. L. Rolston, Phys. Rev. Lett. 83, 4776 (1999).

${ }^{25}$ T. C. Killian, T. Pattard, T. Pohl, and J. M. Rost, Phys. Rep. 449, 77 (2007).

${ }^{26}$ S. Kulin, T. C. Killian, S. D. Bergeson, and S. L. Rolston, Phys. Rev. Lett. 85, 318 (2000).

${ }^{27}$ S. Laha, P. Gupta, C. E. Simien, H. Gao, J. Castro, and T. C. Killian, Phys. Rev. Lett. 99, 155001 (2007).

${ }^{28}$ J. Castro, P. McQuillen, and T. C. Killian, Phys. Rev. Lett. 105, 065004 (2010).

${ }^{29}$ T. C. Killian, P. McQuillen, T. M. O’Neil, and J. Castro, Phys. Plasmas 19, 055701 (2012).

${ }^{30}$ P. McQuillen, J. Castro, T. Strickler, S. J. Bradshaw, and T. C. Killian, Phys. Plasmas 20, 043516 (2013).

${ }^{31}$ S. J. Bradshaw and H. E. Mason, Astro. Astrophys. 407, 1127 (2003).

${ }^{32}$ S. J. Bradshaw and J. A. Klimchuk, Astrophys. J. Suppl. Ser. 194, 26 (2011).

${ }^{33}$ C. E. Simien, Y. C. Chen, P. Gupta, S. Laha, Y. N. Martinez, P. G. Mickelson, S. B. Nagel, and T. C. Killian, Phys. Rev. Lett. 92, 143001 (2004).

${ }^{34}$ M. S. Murillo, Phys. Rev. Lett. 87, 115003 (2001).

${ }^{35}$ Y. C. Chen, C. E. Simien, S. Laha, P. Gupta, Y. N. Martinez, P. G. Mickelson, S. B. Nagel, and T. C. Killian, Phys. Rev. Lett. 93, 265003 (2004).

${ }^{36}$ S. Ichimaru, Statistical Plasma Physics, Volume II: Condensed Plasmas, Frontiers in Physics (Westview Press, Boulder, CO, 2004).

${ }^{37}$ D. B. Melrose, Instabilities in Space and Laboratory Plasmas (Cambridge University Press, Cambridge, 1986).

${ }^{38}$ Waves and Instabilities in Plasmas, edited by F. Cap (Springer-Verlag, Wien, 1994).

${ }^{39}$ S. P. Gary and N. Omidi, J. Plasma Phys. 37, 45 (1987).

${ }^{40}$ D. Grésillon and F. Doveil, Phys. Rev. Lett. 34, 77 (1975).

${ }^{41}$ Y. Nakamura, Y. Nomura, and T. Itoh, Phys. Rev. Lett. 39, 1622 (1977)

${ }^{42}$ Y. Nakamura, H. Bailung, and R. Ichiki, Phys. Plasmas 11, 3795 (2004).

${ }^{43}$ A. Sarma, Y. Saitou, R. Schrittwieser, and Y. Nakamura, Plasma Phys Controlled Fusion 47, 1415 (2005).

${ }^{44}$ J. Castro, H. Gao, and T. C. Killian, Plasma Phys. Controlled Fusion 50, 124011 (2008).

${ }^{45}$ J. Huba, United States Office of Naval Research, and Naval Research Laboratory (U.S.), NRL Plasma Formulary, NRL publication (Naval Research Laboratory, 1998).

${ }^{46}$ F. Robicheaux and J. D. Hanson, Phys. Plasmas 10, 2217 (2003).

${ }^{47}$ E. A. Cummings, J. E. Daily, D. S. Durfee, and S. D. Bergeson, Phys. Rev. Lett. 95, 235001 (2005).

${ }^{48}$ P. Gupta, S. Laha, C. E. Simien, H. Gao, J. Castro, T. C. Killian, and T. Pohl, Phys. Rev. Lett. 99, 75005 (2007).

${ }^{49}$ T. Peter and J. Meyer-ter Vehn, Phys. Rev. A 43, 1998 (1991).

${ }^{50}$ L. S. Brown, D. L. Preston, and R. L. Singleton, Jr., Phys. Rep. 410, 237 (2005).

${ }^{51}$ B. Tashev, F. Baimbetov, C. Deutsch, and P. Fromy, Phys. Plasmas 15, 102701 (2008).

${ }^{52}$ J. N. Glosli, F. R. Graziani, R. M. More, M. S. Murillo, F. H. Streitz, M. P. Surh, L. X. Benedict, S. Hau-Riege, A. B. Langdon, and R. A. London, Phys. Rev. E 78, 025401R (2008).

${ }^{53}$ R. P. Drake, K. G. Estabrook, H. A. Baldis, S. H. Batha, K. S. Bradley, and R. J. Procassini, Phys. Rev. Lett. 73, 1251 (1994).

${ }^{54}$ Q. Z. Yu, Y. T. Li, S. M. Weng, Q. L. Dong, F. Liu, Z. Zhang, J. Zhao, X. Lu, C. Danson, D. Pepler et al., Phys. Plasmas 17, 043106 (2010).

${ }^{55}$ O. Larroche, Phys. Fluids B 5, 2816 (1993).

${ }^{56}$ Y. K. Huang, A Short Treatise on Shock Waves and Equations of State (Nova Science Publishers, Inc., Haupaugge, New York, 2002). 